\title{
Towards Guidance of Electrophysiological Procedures with Real-Time 3D Intracardiac Echocardiography Fusion to C-arm CT
}

\author{
Wolfgang Wein ${ }^{1}$, Estelle Camus ${ }^{2}$, Matthias John ${ }^{3}$, Mamadou Diallo ${ }^{1}$,
} Christophe Duong ${ }^{2}$, Amin Al-Ahmad ${ }^{4}$, Rebecca Fahrig ${ }^{4}$, Ali Khamene ${ }^{1}$, and Chenyang $\mathrm{Xu}^{1}$

1 Imaging \& Visualization Dept., Siemens Corporate Research, Princeton, NJ, USA

2 Siemens Healthcare, Ultrasound Business Unit, Mountain View, CA, USA

3 Siemens Healthcare, AX Business Unit, Forchheim, Germany

${ }^{4}$ Department of Radiology, Stanford University, CA, USA

\begin{abstract}
This paper describes a novel method for improving the navigation and guidance of devices and catheters in electrophysiology and interventional cardiology procedures using volumetric data fusion. The clinical workflow includes the acquisition and reconstruction of CT data from a C-arm X-ray angiographic system and the real-time acquisition of volumetric ultrasound datasets with a new intracardiac real-time $3 \mathrm{D}$ ultrasound catheter. Mono- and multi-modal volumetric registration methods, as well as visualization modes, that are suitable for real-time fusion are described, which are the key components of this work. Evaluation on phantom and in-vivo animal data shows that it is feasible to register and track the motion of real-time 3D intracardiac ultrasound in C-arm CT.
\end{abstract}

\section{Introduction}

Pre- and intra-procedural imaging is an integral part of many cardiac interventions, both surgical and minimally invasive (percutaneous) ones. Regarding high-resolution static pre- and intra-operative imaging, Computed Tomography (CT), including C-arm CT, is most widely used for diagnosis and planning. However these imaging modalities do not have real-time capabilities. As a real-time interventional modality, intra-cardiac echocardiography (ICE) is now widely used in many cathlabs, e.g. for guidance of left atrial ablation procedures.

We have developed an intra-cardiac ultrasound catheter (Siemens ACUSON AcuNav $^{\mathrm{TM}} \mathrm{V}$ ), which is capable of acquiring ultrasound volumes from within the heart in real-time [1]. This novel imaging modality may, on its own, have an tremendous impact on intra-cardiac procedures. However, even 2D ICE technology, when used in the electrophysiology (EP) environment, has been found to require adequate training and experience in order to fully exploit its benefits. Finding the orientation of the ICE catheter within the body and recognizing anatomical structures can be challenging, especially when the catheter is moved quickly. The 3D capability of our new system allows fusion of real-time ICE 


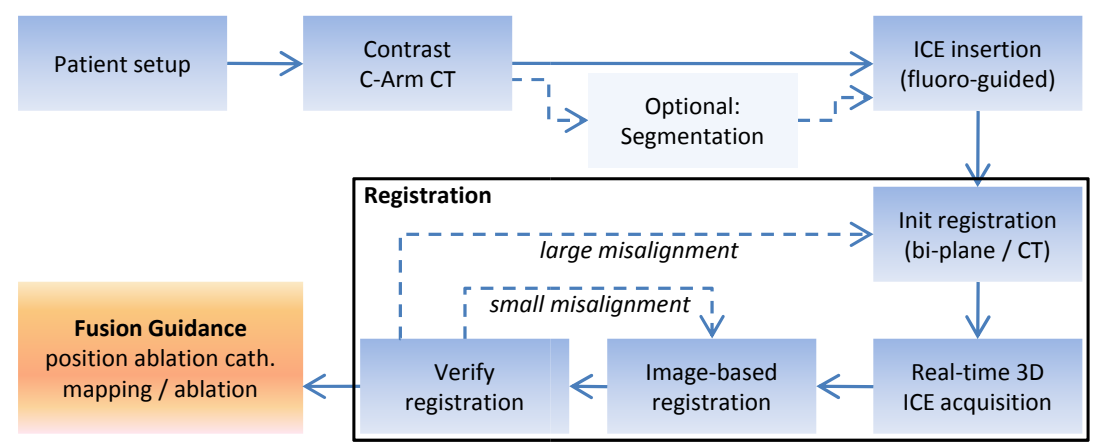

Fig. 1. Workflow overview

data with pre-operative or intra-operative high-resolution CT or MRI images, potentially reducing procedure time and improving treatment outcome. Baseline anatomical images can be combined with ICE imaging, which provides real-time monitoring of morphological and functional changes of cardiac tissue.

The CartoSound module (Biosense Webster, Israel) allows to superimpose 2D real-time ICE with an integrated magnetic position sensor to pre-operative information. Related work regarding registration and overall fusion setup is described in 2 (using heart surface points as registration features), and 3. In the latter, an ECG- and respiration-gated ICE sweep is registered to CT, by manual initialization and image-based refinement. Thereafter, the position sensor update allows for live fusion of the 2D ICE with superimposed CT slices and segmented structures.

In our work, we would like to take advantage of the capabilities of the novel real-time 3D ICE catheter. In order to minimize set-up time, increase flexibility, and ensure compatibility to any EP mapping solution used in clinical practice we target a purely image-based technique for registration.

\section{Methods}

\subsection{Proposed Clinical Workflow}

In the following, we assume the case of an ablation procedure to treat atrial fibrillation. The proposed workflow (as outlined in figure 1) can also be used e.g. for guidance of transseptal punctures or guidance of intracorporeal devices during cardiac interventions.

At the beginning of the procedure, a baseline cardiac C-arm CT reconstruction with contrast agent injection is performed. Optionally, automatic or semiautomatic segmentation of the heart chambers (in particular of the left atrium) and the esophagus may be performed. Then, the ICE catheter is inserted into the body and guided to the heart (typically to the right side via the femoral or jugular vein), if needed, under fluoroscopic guidance. The ICE-CT registration 
is initialized by locating the ICE catheter tip either in a bi-plane fluoro view, or in a low-dose C-arm CT reconstruction. This data is in turn automatically registered to the baseline volume. Next, an ICE acquisition is performed, typically by rotating the ICE catheter such that it covers the whole left atrium. This recording is automatically registered to the baseline CT, using the algorithms described below. After visual assessment, this process can be repeated depending on the quality of the alignment. Thereafter, the actual EP procedure, which includes electromapping and ablation can be executed under fusion imaging. The position of the ICE information within the C-arm CT volume can be updated any time by image-based tracking and/or additional fluoroscopic acquisitions.

In the next section, we describe algorithms supporting this workflow by providing image-based registration and tracking of real-time 3D ICE within C-arm CT.

\subsection{Cardiac Phase Detection}

Since the baseline CT data is only available in one cardiac phase (e.g. enddiastole), only volumes of the live ICE data corresponding to that phase can be considered for registration. ECG gating is a common solution; however the ECG signal might be delayed with respect to actual cardiac motion, or, in the worst case, not directly be related at all, in patients with heart arrhythmia. An imagebased technique for detecting periodic motion has therefore been chosen that uses extrema of the cumulated phase shift in the spectral domain of successive image frames [4. End-diastole ICE frames are tagged with this method and then used for image-based registration to the baseline data 1 .

\subsection{Registration of Single Volume}

In order to allow image-based registration of ICE to C-arm CT, the modalities have to be made more similar in a pre-processing step, and then a nonlinear optimization refines the transformation parameters with respect to a measure of the alignment quality. For CT-ultrasound registration in the abdomen, the stateof-the-art uses a simulation of ultrasonic echogenicity and reflection from CT, and a specific similarity metric to overcome their unknown combination [5]. In the case of echocardiography, only the reflection term is significant, showing the myocardial walls of the cardiac cavities. The blood inside the chambers results in completely hypoechoic regions. In addition, the diffusivity of the reflections at cardiac walls is relatively high, and shadowing does not usually occur within our anatomy of interest (i.e. imaging the left atrium from the right atrium). We therefore drop the simulated echogenicity and orientation-dependency from the approach in [5], and precompute a global simulated ultrasound reflection volume, henceforth denoted $R$, from the baseline C-arm CT volume. The registration of a single end-diastole ICE volume $U$ to the baseline volume is then described as

$$
\arg \max _{\phi} \mathrm{CC}\left(U, R \circ T^{-1}(\phi)\right)
$$

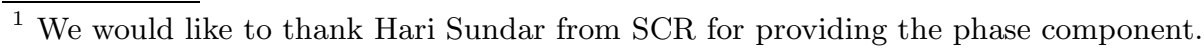


where $T$ is the rigid homogeneous transformation matrix mapping $U$ on $R$, parameterized by a 6 -vector $\phi$. CC is the Pearson's correlation coefficient, which we use because it is independent of scale and bias between the simulated reflection and actual ultrasound intensities. $R \circ T^{-1}$ denotes a resampling of $R$ with the transformation $T^{-1}$. The scan lines of the ICE data are scan converted into a cartesian grid, which both serves as a low-pass filter and makes the resolution more isotropic.

An initialization is provided by manually extracting the position of the ICE catheter from a bi-plane fluoroscopic acquisition, or a low-dose C-arm CT reconstruction. The optimization then consists of a global search on the rotation around the catheter axis, followed by a Simplex direct search method.

\subsection{Trajectory Registration}

A single ICE volume provides a limited field of view within the cardiac anatomy, potentially resulting in multiple ambiguous locations suggesting good alignment. Because the physician is maneuvering the ICE catheter within the heart to image different anatomical regions of interest, successive end-diastole ICE volumes can be used to create an extended field-of-view volume. These volumes need to be brought into correct alignment themselves. We simultaneously optimize monoand multi-modal similarity metrics for that purpose. Now the pose parameters of a sequence of successive ICE frames are refined, until both the alignment to the baseline $\mathrm{C}$-arm $\mathrm{CT}$, as well as their internal alignment are optimal:

$$
\arg \max _{\phi} \frac{1}{N} \sum_{i=1}^{N} \operatorname{CC}\left(U_{i}, R \circ T_{i}^{-1}\right)-\frac{\alpha}{N-1} \sum_{i=1}^{N-1} \operatorname{SAD}\left(U_{i}, U_{i+1} \circ\left(T_{i}^{-1} T_{i+1}\right)\right)
$$

As before, the correlation coefficient serves as multimodal measure. Sum of Absolute Differences $\operatorname{SAD}(U, V)=\frac{1}{|\Omega|} \sum\left|u_{i}-v_{i}\right|$ works best for measuring the alignment of successive ICE volumes, where the flexibility of CC is not needed. Since it is a dissimilarity measure, we negate and weight it with a parameter $\alpha$. Rather than optimizing the unconstrained problem with $N * 6$ degrees of freedom (DOF), we manipulate only the first and last transformation, assuming all volumes are located on a linear trajectory:

$$
\phi=\left(\begin{array}{c}
\phi_{0} \\
\phi_{1}
\end{array}\right) ; \quad T_{i}=T\left(\phi_{0}\right) \exp \left(\frac{i-1}{N-1} \log \left(T\left(\phi_{0}\right)^{-1} T\left(\phi_{1}\right)\right)\right)
$$

Here, Lie manifold based interpolation of transformation matrices is used; the optimization problem is reduced to 12 DOF. Note that we do not have to weight the individual SAD values with respect to volume overlap, because by using this manifold interpolation all successive volumes will have equal overlap.

For arbitrarily long ICE sequences, or continuous motion tracking, the registration described by equation 3 , is repeatedly applied to an overlapping subset of all volumes. 


\subsection{Implementation Details}

We use a software framework developed in $\mathrm{C}++$, OpenGL and GLSL. As is common nowadays, we have accelerated scan-conversion, volume resampling, similarity measure computation, and visualization, by implementing them on a graphics processing unit (GPU). The incoming ICE volumes are immediately uploaded to a ringbuffer of 3D textures in graphics memory.

The registration of a single catheter motion with $10 \mathrm{ICE}$ volumes takes $\approx 0.6 \mathrm{~s}$ (where each ICE volume is used with $\approx 130 \mathrm{~K}$ voxels) on an Nvidia GeForce 8800 GTX GPU, compared to $27 s$ in a reference software implementation.

For enhancing the visual perception of the ICE data, an efficient shading technique is used for volume rendering, that does not require 3D volume gradients 6]. The real-time visualization comprises three blended multi-planar reformatted (MPR) slices of the C-arm CT volume and ICE superimposed in color, cropped $3 \mathrm{D}$ volume rendering, as well as mesh visualization of an optional four-chamber segmentation of the heart, see figure 4 (b).

\section{Experiments}

\subsection{Phantom Data}

We conducted phantom experiments with the Siemens ACUSON AcuNav V ICE catheter submerged in an acrylic water container, which was cushioned with rubber material to minimize acoustic reflections at the walls. It was placed on the patient table of a Siemens Axiom Artis angiographic suite, such that we could acquire 3D C-arm CT (software: syngo@ DynaCT Cardiac) at any time. We attached a magnetic tracking sensor (Ascension MicroBird) to the ICE catheter and recorded both static and dynamic sequences on clay phantoms (a left atrium model and a small Halloween pumpkin, the latter chosen because of its sharp facial imaging features, but also seasonally inspired).

In order to use the magnetic tracking information, spatial calibration has to be performed to derive the location of the actual ICE catheter image with respect to the tracking sensor's coordinate system. Because of the unique imaging characteristics of the novel real-time 3D ICE catheter none of the off-the-shelf calibration techniques seemed appropriate. We decided to use a simultaneous optimization of the calibration pose $\phi_{C}$ and registration $\phi_{R}$, comparing 12 ICE volumes from a wide sweep across the clay objects against the $\mathrm{C}$-arm CT volume:

$$
\arg \max _{\phi} \sum_{i=1}^{N} \mathrm{CC}\left(U_{i}, R \circ\left(T_{R}\left(\phi_{1}\right) T_{T}^{i} T_{C}\left(\phi_{0}\right)\right)^{-1}\right) ; \quad \phi=\left(\begin{array}{c}
\phi_{C} \\
\phi_{R}
\end{array}\right)
$$

where $T_{C}\left(\phi_{C}\right)$ is the calibration transformation (ICE to sensor), $T_{T}^{i}$ the tracking matrix (sensor to tracker reference), and $T_{R}\left(\phi_{R}\right)$ the registration transformation (tracker reference to $\mathrm{CT}$ ). $R$ is here replaced with the original C-arm CT volume, because for such water/clay data the CT measurements correspond directly to ultrasonic reflection. We verifed the calibration with a follow-up C-arm CT of 


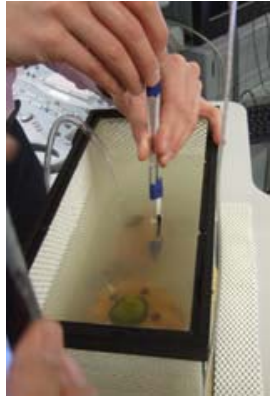

(a) Setup

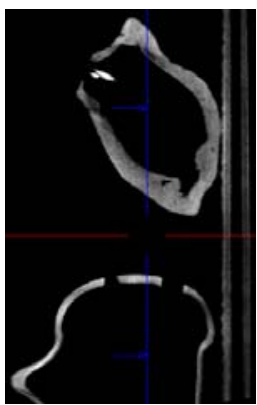

(b) CT

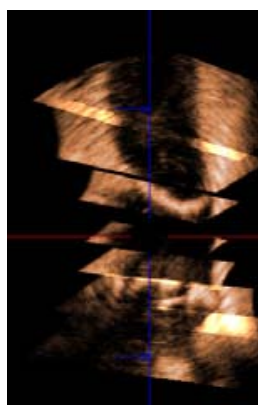

(c) ICE

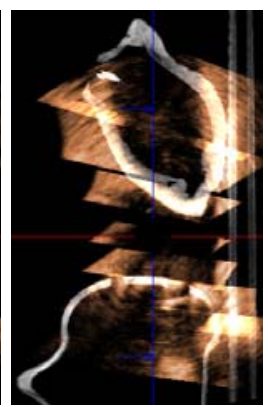

(d) Overlay

Fig. 2. Evaluation on phantom data

the ICE catheter mounted in a still configuration. The result allowed us to depict both static and dynamic real-time 3D ICE sequences fused with the respective Carm CT data (figure 2). We then evaluated the trajectory registration method using the tracked and calibrated ICE as Ground Truth, on 18 volumes from another sweep over both clay objects. For many algorithm executions, using $7-12$ ICE volumes at a time for minimizing equation 3 , advancing $1-4$ volumes in between, resulted in a mean accuracy in translation and rotation of $2.2 \mathrm{~mm}$ and $1.8^{\circ}$ respectively over the entire motion. The weighting $\alpha$ interestingly has minor influence, as long as it is not chosen to be extremely large. In that case, the multi-modal component is ignored, and the registration of successive frames creates a drift (error at last frame $7.4 \mathrm{~mm}, 7.4^{\circ}$ ).

\subsection{Animal Studies}

We are currently evaluating the real-time 3D ICE imaging modality itself and our approach for ICE-CT fusion (works in progress) in a series of animal and human studies. In the following, we report on initial results from data acquired during an acute pig study2.

The baseline C-arm CT reconstruction was executed in forced breath-hold and cardiac gating. All ICE recordings were done during normal respiration. The used ICE catheter did not have a position sensor integrated in its housing. Therefore, we defined quasi-ground truth registration on the recorded ICE sequences, using all the advanced visualization at our disposal (figure 4). Figure 3 depicts a comparison of physician-defined ground truth and the result of our image-based registration (section 2.3). For four data sets (showing aortic valve, left atrium, left ventricle), the average discrepancy between expert-defined and image-based registration was $16 \mathrm{~mm}$ and $11^{\circ}$.

We also evaluated the trajectory registration on a recording where the ICE catheter was rotated within the right atrium. The first volume was roughly aligned by a physician, and the rotation direction of the trajectory indicated by

${ }^{2}$ The procedure was performed on a $35-\mathrm{kg}$ pig according to a protocol approved by Stanford University's Institutional Animal Care and Use Committee. 


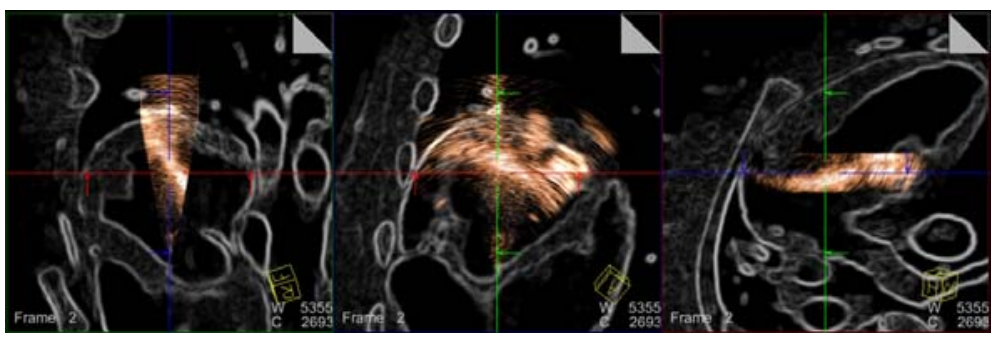

(a) Ground Truth

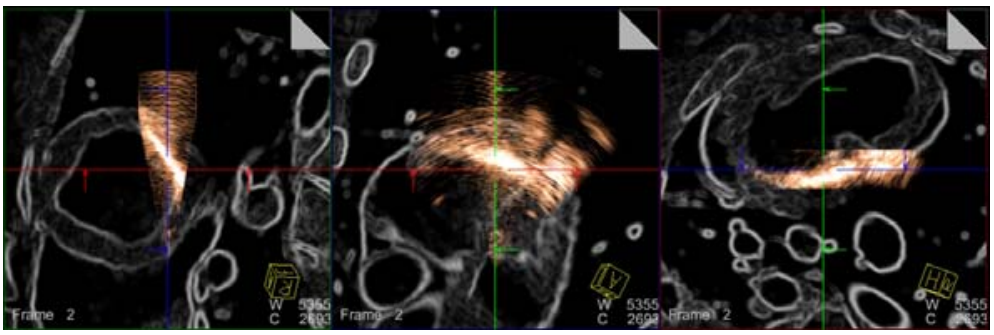

(b) after automatic registration

Fig. 3. Cross-sections of CT reflection overlayed with single end-diastole ICE volume

manually dragging the series of end-diastole volumes. The automatic registration then nicely pulls the whole sequence into good visual alignment, see figure 4(a).

\section{Conclusion}

We presented the first image fusion results with a novel real-time 3D intra-cardiac ultrasound catheter. We showed that automatic registration to C-arm CT data, and appropriate fused visualization for guiding EP procedures, are possible, after a rough registration initialization. The underlying technological novelties are the use of an image-based detection of cardiac phase, an algorithm for simultaneous mono- and multi-modal registration of a trajectory of ICE volumes, and dedicated real-time visualization. Furthermore, we detailed a workflow, which could enable such a fusion system to be used in clinical practice in the future, with all necessary imaging integrated in the interventional suite.

We evaluated our methods quantitatively on phantom data, and qualitatively on in-vivo pig data. The trajectory registration technique can be used to track cardiac end-diastole ultrasound volumes within a C-arm CT volume in realtime, without additional position tracking hardware. A particular advantage is that our registration is directly based on the anatomical structures in the two modalities, which inherently compensates for residual cardiac and respiratory motion. Given the limited field of view offered by the ICE catheter, accurate registration of 3D ICE volumes with $\mathrm{CT}$ datasets is a difficult task. Therefore, an extended-field-of-view acquisition, as proposed, is crucial for non-ambiguous, more accurate alignment. 


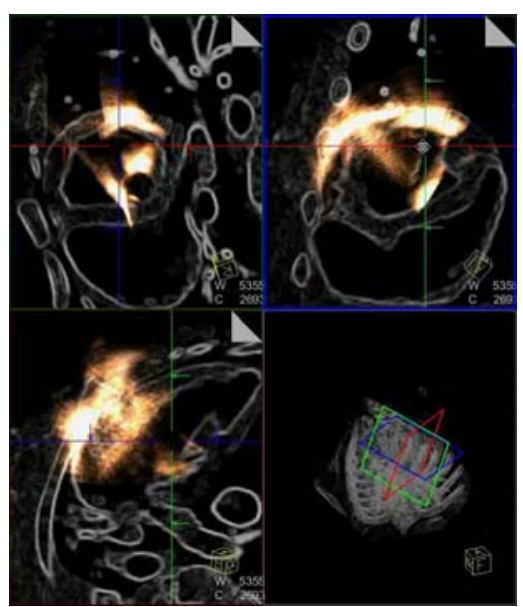

(a) Trajectory Registration

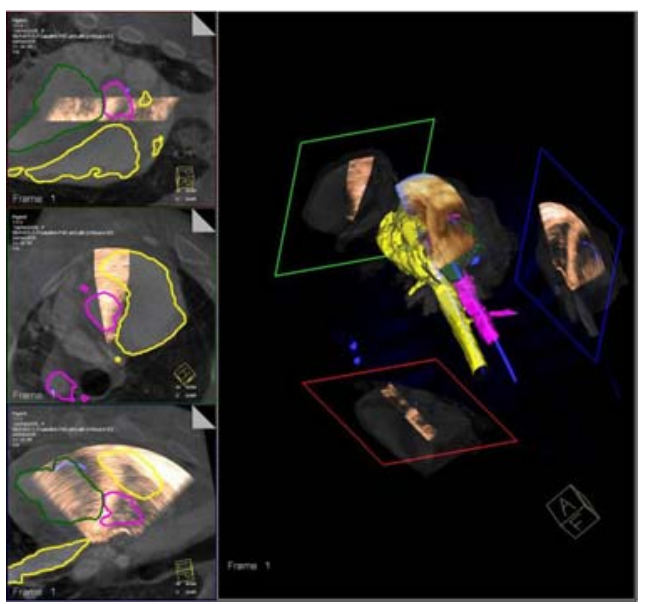

(b) 3D Visualization

Fig. 4. Left: Trajectory registration result of a rotation in a pig's left atrium, with ICE (yellow) superimposed onto the CT gradient volume in $3 \mathrm{MPRs}$, and their corresponding location in 3D. Right: 3D visualization of an ICE volume with C-arm CT and outlines from a four-chamber segmentation (with $3 \mathrm{MPRs}$ in the left column).

Further experimental investigations are needed in order to create proper ground truth registration and refine our method in terms of robust, contiguous tracking anywhere in the heart. Particularly, the limitations of the concept of a linear motion trajectory needs to be assessed more precisely in the case of the ICE catheter, and could be addressed with more powerful motion models.

\section{References}

1. Wilser, W., Barnes, S., Garbini, L.: Helical acoustic array for medical ultrasound. United States Patent No. 20,080,125,659 (May 2008)

2. Zhong, H., Kanade, T., Schwartzman, D.: Virtual touch: An efficient registration method for catheter navigation in left atrium. In: MICCAI 2006 proceedings (2006)

3. Sun, Y., Kadoury, S., Li, Y., John, M., Resnick, J., Plambeck, G., Liao, R., Sauer, F., Xu, C.: Image guidance of intracardiac ultrasound with fusion of pre-operative images. In: Ayache, N., Ourselin, S., Maeder, A. (eds.) MICCAI 2007, Part I. LNCS, vol. 4791, pp. 60-67. Springer, Heidelberg (2007)

4. Sundar, H., Khamene, A., Yatziv, L., Wein, W., Xu, C.: Automatic image-based cardiac and respiratory cycle synchronization and gating of image sequences. In: Yang, G.-Z., et al. (eds.) MICCAI 2009, Part I. LNCS, vol. 5761, pp. 381-388. Springer, Heidelberg (2009)

5. Wein, W., Brunke, S., Khamene, A., Callstrom, M., Navab, N.: Automatic ctultrasound registration for diagnostic imaging and image-guided intervention. Medical Image Analysis 12, 577-585 (2008)

6. Sumanaweera, T.: Applying real-time shading to 3D ultrasound visualization. GPU Gems, ch. 40 (2004) 